\title{
TECNOLOGÍA DE HOJAS LÍTICAS EN TIERRAS ALTAS ANDINAS: PERspectivas DESDE LA PUNA DE LA PROVINCIA DE SALTA
}

(Argentina)

\author{
Federico Restifo ${ }^{1}$
}

\section{* Introducción}

\section{Resumen}

Presentamos un estudio sobre la tecnología de hojas líticas de la Puna de Salta, Argentina, en base tomando como base dos áreas: la cuenca de Pastos Grandes y el valle de San Antonio de los Cobres, distantes $60 \mathrm{~km}$ entre sí. En primer lugar nos focalizamos en la cronología y la aparición de la tecnología de hojas líticos, y luego en su dispersión espacial. Para el primer caso analizamos diferentes atributos de artefactos formatizados, desechos de talla y núcleos desde una perspectiva macroscópica. Para el segundo caso comparamos muestras de artefactos formatizados sobre hojas - lanceolados unifaciales - con base en un análisis estadístico de sus variables métricas. Los sitios considerados son Alero Cuevas, con una secuencia extendida a lo largo del Holoceno,

y Ramadas Perfil Norte, con fechados de fines del Holoceno Medio. Nuestros resultados apoyan la idea de la aparición de la tecnología de hojas hacia los ca. $5200 \mathrm{AP}$, persistiendo al menos hasta los ca. 4200 AP. Asimismo, sugerimos que su dispersión espacial pudo haber ocurrido a partir de la circulación de información en el espacio, implicando posibles contactos entre grupos distantes. Finalmente, discutimos estos resultados considerando la puna argentina y el norte de Chile.

Palabras claves: Puna - Salta - Hojas Líticas - Holoceno Medio.

Abstract

We present a study on blade technology of the Puna of Salta, Argentina, based on two study areas: the Pastos Grandes Basin and the Valley of San Antonio de los Cobres, located $60 \mathrm{~km}$ apart from each other. First we focus on the timing of the onset of blade technology, and then its spatial dispersion. For the first case we analyze different attributes of shaped artifacts, debitage and cores from a macroscopic perspective. For the second case we compare shaped artifact samples on blade blanks-unifacial lanceolated- based on a statistical analysis of their metric variables. The sites considered are Alero Cuevas, with an extended sequence throughout the Holocene, and Ramadas Profile Norte,

dating to the end of the Middle Holocene. Our results support the idea of the emergence of blade technology to ca. $5200 \mathrm{BP}$, persisting at least until ca. $4200 \mathrm{BP}$. We also suggest that spatial dispersion could have occurred from the flow of information in space, implying possible contacts between distant groups. Finally, we consider these results for the Argentine Puna and Northern Chile regions.

Key words: Puna - Salta - Blades - Middle Holocene. Recibido: Abril 2014. Aceptado: Octubre 2015
Uno de los cambios más notorios en tecnología lítica, registrados en diferentes contextos arqueológicos de la puna argentina, es la aparición de la tecnología de hojas (Fernández 1971, 1983; Schobinger et al. 1974; Fernández Distel 1978, 2007). Tanto sus productos en términos de formas base y artefactos formatizados, incluyendo las típicas morfologías alargadas, así como su asociación con clases de desechos de talla y núcleos particulares, constituyen señales distintivas de esta alternativa tecnológica (Boëda 1997; Bar-Yosef y Kuhn 1999).

La tecnología de hojas en la puna argentina se vincula con la denominada "Industria Saladillense" (Fernández 1971), definida en la Puna de Jujuy, con en base a materiales del área de Salinas Grandes. Un rasgo particular del conjunto de artefactos asociados es la presencia de las denominadas "Puntas Saladillo". Se trata de una clase de artefacto que reúne regularidades tecnológicas tales como el limbo lanceolado, la unifacialidad predominante - pudiendo presentar retoques en la cara ventral que reducen el espesor del bulbo-, el uso de hojas o lascas de módulo laminar como forma base, y un mayor grado de estandarización (Fernández 1971 [lámina XLVII], 1983; Fernández Distel 1978). Asimismo, en el marco de la arqueología de la Puna de Salta, estos artefactos también han sido denominados "lanceolados unifaciales", terminología que retomamos en este trabajo (López 2008). Por su parte, en la Puna de Catamarca aún no se han registrado evidencias de tecnología de hojas (Aschero y Hocsman 2011).

Si bien en Salinas Grandes esta clase de artefacto proviene de contextos de superficie, con base en características geomorfológicas del terreno fue asociado a fechados de

1 Consejo Nacional de Investigaciones Científicas y Técnicas, Instituto de Arqueología, Facultad Filosofía y Letras, Universidad de Buenos Aires. 25 de Mayo 217 (1002), $3^{\circ}$ Piso, Ciudad Autónoma de Buenos Aires, ARGENTINA. Email: federicorestifo@gmail.com 


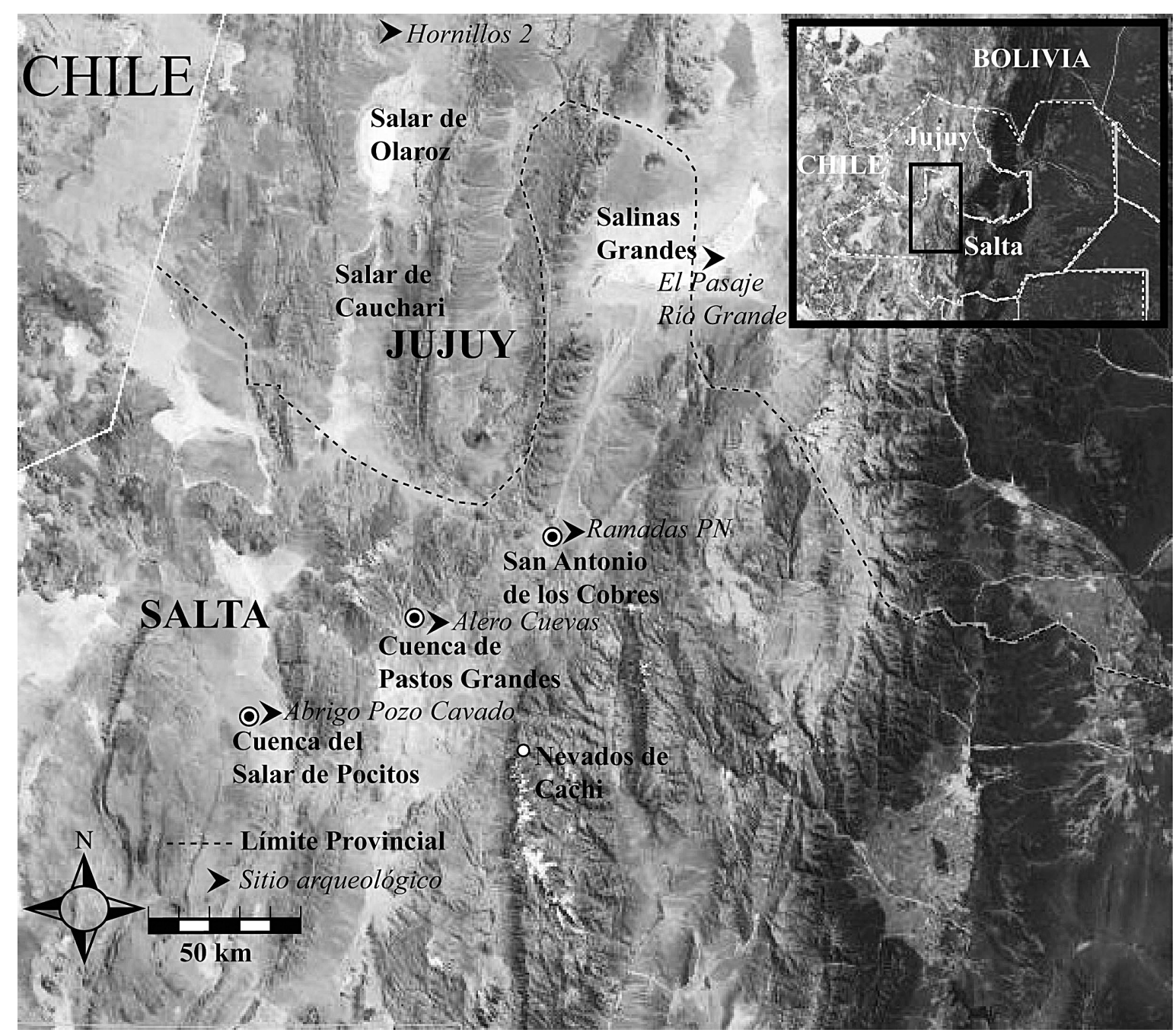

Figura 1. Mapa con detalle de sectores de tierras altas de las Provincias de Salta y Jujuy, y sitios arqueológicos con fechados radiocarbónicas y presencia de artefactos vinculados con tecnología de hojas.

momentos de fines del Holoceno Medio y comienzos del Holoceno Tardío. Esto se determinó principalmente a partir de dos fechados, uno de $3995 \pm 75 \mathrm{AP}$, correspondiente al sitio "El Pasaje" (Fernández Distel 2007), y otro de $5520 \pm 270$ AP proveniente del sitio "Río Grande" (Fernández 1983) (Figura 1). Asimismo, en el norte de Chile también se ha registrado el incremento de hojas líticas hacia ca. $5000 \mathrm{AP}$, especialmente a partir de la evidencia de sitios de las zonas de Tulán y Puripica (Núñez et al. 2006). Por su parte, en los contextos arqueológicos del Holoceno Temprano e inicios del Holoceno Medio de la puna argentina predomina la tecnología de lascas, sin un patrón definido en cuanto a morfología (Restifo y Hoguin 2012). En este sentido, si bien el sitio Hornillos 2 (Puna de Jujuy) presenta evidencia del uso de hojas como forma base de artefactos formatizados desde fines del Holoceno Temprano y hasta el 6200 AP (final de la secuencia), sus frecuencias son bajas (Hoguin 2013) (Figura 1). Entonces, los antecedentes sugieren que el empleo de hojas líticas comienza a cobrar mayor visibilidad en el registro arqueológico de la puna una vez avanzado el Holoceno Medio.

Con base en estos antecedentes, proponemos como primer objetivo aportar a la discusión de la aparición de la tecnología de hojas en la puna argentina a partir del caso 


\begin{tabular}{|l|l|l|l|l|}
\hline Sitio & Laboratorio & Fechado AP & Calibración 2 sigmas AC-DC & Material \\
\hline Alero Cuevas AC-C2-F2 & LP-1655 & $4210 \pm 70$ & 2930 AC-2530 AC & Óseo \\
\hline Alero Cuevas AC-C7-F2 & AA-90383 & $5106 \pm 68$ & 4050 AC-3710 AC & Carbón \\
\hline Alero Cuevas AC-C1-F3 & AA-71137 & $6506 \pm 58$ & 5610 AC-5340 AC & Óseo \\
\hline Alero Cuevas AC-C2-F3 & LP-1759 & $6510 \pm 80$ & 5620 AC-5320 AC & Carbón \\
\hline Alero Cuevas AC-C2-F4 & AA-71135 & $8504 \pm 52$ & 7600 AC-7490 AC & Carbón \\
\hline Alero Cuevas AC-C2-F4 & AA-71136 & $8838 \pm 52$ & 8210 AC-7750 AC & Carbón \\
\hline Alero Cuevas AC-C4-F4 & LP-1736 & $9650 \pm 100$ & 9300 AC-8750 AC & Carbón \\
\hline Ramadas PN capa C & UGA-8726 & $5210 \pm 40$ & 4220 AC-3950 AC & Turba \\
\hline Ramadas PN capa C & AA-95944 & $5273 \pm 71$ & $4230-3996$ AC & Óseo \\
\hline Ramadas PN capa C & AA-95943 & $5333 \pm 40$ & $4225-3959$ AC & Óseo \\
\hline
\end{tabular}

Tabla 1. Fechados correspondientes al Holoceno Temprano y Medio en sitios arqueológicos de la cuenca de Pastos Grandes y el valle de San Anto nio de los Cobres.

particular de la Puna de Salta, y focalizando en las investigaciones desarrolladas en la cuenca de Pastos Grandes (López 2008) y el valle de San Antonio de los Cobres (Muscio 2004), distantes entre sí $60 \mathrm{~km}$. Respecto de la primer área se consideró el sitio Alero Cuevas, el cual posee una secuencia arqueológica extendida a lo largo del Holoceno (Tabla 1). En relación a la segunda área, se tomó en cuenta el sitio Ramadas Perfil Norte, con fechados correspondientes al Holoceno Medio (Tabla 1).

A su vez, el segundo objetivo consiste en abordar de manera preliminar el fenómeno de la dispersión espacial de la tecnología de hojas líticas, específicamente a partir de los artefactos lanceolados unifaciales. De este modo, aparte de considerar aquellos artefactos recuperados en los sitios mencionados, también incluimos aquellos obtenidos en contextos de superficie de ambas áreas. La distribución de una misma clase de artefacto en el espacio, y sus semejanzas métricas, puede ser indicador de circulación de información y posibles interacciones entre poblaciones humanas. En el marco de la arqueología patagónica, este abordaje cobró relevancia especialmente en los estudios de distribución espacial de las denominadas puntas de proyectil "Bird" (Franco et al. 2010). Del mismo modo, dicho abordaje puede contribuir a una discusión sobre interacciones entre poblaciones humanas de cazadores recolectores de la puna argentina.

El estudio de los materiales se realizó a partir de dos tipos de análisis. En relación al primer objetivo, analizamos diferentes atributos de artefactos formatizados, desechos de talla y núcleos, y en función de la identificación de hojas principalmente. En cuanto al segundo objetivo, se realizó el análisis comparativo de artefactos lanceolados unifaciales de la cuenca de Pastos Grandes (Alero Cuevas y materiales de superficie) y del valle de San Antonio de los Cobres (Ramadas Perfil Norte y materiales de superficie). Para este caso, el análisis realizado fue de base estadística, utilizando variables métricas (Bettinger y Eerkens 1999; Franco et al. 2010). Por último, sobre la base de los resultados obtenidos se apuntó a una discusión considerando la puna argentina y de Chile. A su vez, se tomó en cuenta el marco regional de los procesos de cambio cultural, como es el caso del proceso de intensificación en la explotación de camélidos (Yacobaccio 2001; Cartajena et al. 2007).

\section{Aspectos generales sobre la tecnología de hojas}

Siguiendo a diferentes autores, cuando hablamos de hojas líticas hacemos referencia a artefactos que presentan un patrón de atributos específicos distintivos, tanto por sus dimensiones de longitud y ancho como por los atributos de las caras (Aschero 1975; Boëda 1997; Bar-Yosef y Kuhn 1999; Kuhn 2006). Así, destacamos la morfología alargada, caracterizada por una relación en la que la longitud corresponde al doble de la medida del ancho o más. Para expresar esta relación recurrimos al cálculo del módulo de longitud/anchura (ver apartado de metodología). A su vez, destacamos la presencia de bordes rectos paralelos o subparalelos, y de aristas rectas paralelas o subparalelas en cara dorsal, lo que indica extracciones 
anteriores. En general, el eje técnico, entendido como aquel que divide en dos partes iguales al talón, se presenta de manera paralela a los negativos de extracción y aristas.

Estos rasgos recurrentes que caracterizan a las hojas se diferencian de aquello a lo que denominamos "lascas" (Tixier 1963). Con dicho término aludimos a piezas que presentan un patrón de mayor variación, en términos de su relación entre longitud y ancho, así como de la disposición de las aristas y bordes y de la dirección del eje técnico en función de estas, últimas, pudiendo ser transversal, oblicuo, etc. Si bien las "lascas" pueden presentar una medida de longitud que supere en dos veces o más al ancho, los rasgos especificados correspondientes a la cara dorsal, así como la disposición del eje técnico serán determinantes a la hora de diferenciarlas de las hojas.

Otro aspecto relevante es la identificación de núcleos asociados, con evidencia de extracciones laminares, lo que contribuye a la comprensión de lo que denominamos un sistema tecnológico de hojas (Aschero 1975; Boëda 1997). En este sentido, la obtención de hojas demanda la preparación de núcleos particulares, implicando, por ejemplo, la generación de una plataforma y una arista específica para la primera extracción, y dando lugar a morfologías particulares como las prismáticas o piramidales (Bellelli y Nami 1994; Quintero y Wilke 1995; Bar-Yosef y Kuhn 1999). Asimismo, las hojas pueden extraerse de un núcleo que no estuvo preparado para tal fin, como podría ser un núcleo de lascas. En este caso, la obtención de las hojas puede darse mediante el aprovechamiento de una arista favorable (Hoguin 2013), pero interrumpiéndose al quedar ésta eliminada, generando en consecuencia escasas hojas a partir de dicho núcleo. En este caso no hablaríamos de tecnología de hojas, puesto que no se trata de un sistema tecnológico orientado a tal fin, sino más bien al aprovechamiento, podríamos decir oportunista, de un núcleo orientado hacia otros fines, en este caso lascas.

\section{El caso de la puna de la provincia de Salta: sitios es- tratificados y de superficie}

La puna argentina constituye una prolongación del denominado Altiplano Andino (Turner 1964; Vilela 1969), el cual se extiende desde el sur de Perú, abarcando también el oeste de Bolivia y sectores del norte de Chile. Confor- ma, a su vez, un ambiente ecológico particular dadas sus características de desierto de altura, superando los 3000 msnm. Entre estas características se destacan la aridez, la baja productividad primaria, la intensa radiación solar, la alta amplitud térmica diaria y la alta variabilidad tanto espacial como temporal en la distribución de recursos críticos para la subsistencia (Ruthsatz y Movia 1975), lo que ocasiona condicionantes para la adaptación humana (Muscio 2004). Dentro de la puna argentina, este trabajo se concentra en dos áreas de la Puna de Salta. Por un lado, la cuenca de Pastos Grandes (cuenca de PG), ubicada a una altura de $4000 \mathrm{msnm}$. Por otro lado, destacamos el valle de San Antonio de los Cobres (valle de SAC), ubicado a una altura de $3700 \mathrm{msnm}$ (Figura 1).

En la cuenca de PG fue localizado el sitio Alero Cuevas, en el año 2004. Tal como lo indica su nombre, se trata de un alero, el cual presenta una línea de goteo de 19,3 m en línea recta, y profundidades variables a la misma, que están entre los 1,25 m y los 8,7 m (López 2008). Se destaca por estar emplazado sobre el faldeo de un cerro en la Quebrada de las Cuevas, a una altura de $4400 \mathrm{msnm}$. Hasta el momento excavamos siete cuadrículas de $1 \mathrm{~m}^{2}$ y dos sondeos de $0,5 \mathrm{~m}^{2}$. El sitio posee una secuencia cronológica que se extiende a lo largo del Holoceno Temprano, Medio y Tardío (López 2008) (Tabla 1). Por este motivo, Alero Cuevas constituye la base cronológica para abordar el estudio de la evolución y el cambio tecnológico en la Puna de Salta, permitiendo abarcar la escala temporal amplia. Los fechados considerados en este trabajo son los que abarcan el segmento de ca. 9650-4000 AP, correspondientes a las capas $\mathrm{F}_{4}, \mathrm{~F}_{3}$ y F2. Si bien el sitio posee fechados para el segmento de ca. 2000-640 AP, no realizamos el análisis de los materiales de las capas correspondientes, dado que fueron tratados en el marco de otro proyecto, enfocado en tecnología lítica de grupos con economías de producción de alimentos (Mercuri 2011). Sin embargo, resultados de dicho proyecto serán mencionados en la discusión.

Por su parte, el sitio Ramadas Perfil Norte se encuentra en el valle de SAC, específicamente en el sector de Fondo de Cuenca. En dicho sector es común la presencia de cárcavas de escurrimiento formadas por los arroyos, cuyas nacientes se ubican en las quebradas laterales. Tales cárcavas exponen perfiles sedimentarios aluviales con materiales arqueológicos. En uno de estos perfiles expuestos 
fue detectado Ramadas Perfil Norte (Ramadas PN, de aquí en adelante) (Muscio 2011). En este sitio se destaca la capa $\mathrm{C}$, que es la que contiene material arqueológico. La misma se ubica por debajo de una capa de diatomita que habría actuado como sello, impidiendo la intrusión de material de superficie. La matriz de la capa $\mathrm{C}$ es de turba fósil, lo que permitió la obtención de un fechado de ca. $5200 \mathrm{AP}$, luego confirmado por otros dos fechados sobre material óseo (Tabla 1). De este modo, Ramadas PN se ubica en el segmento de finales del Holoceno Medio, y a su vez, favorece la comparación con el componente de cronología similar del sitio Alero Cuevas. Hasta el momento excavamos una unidad de $2 \mathrm{~m}$ x $1 \mathrm{~m}$, y un sondeo de $0,5 \mathrm{~m}^{2}$ en el perfil enfrentado (Perfil Sur).

Por último, las prospecciones tanto en la cuenca de PG como en el valle de SAC permitieron detectar concentraciones de material arqueológico en superficie, las que fueron investigadas sistemáticamente mediante transectas (véase Muscio 2004; López 2008). En el caso de la cuenca de PG, destacamos los sitios de superficie de los geoambientes de quebradas y borde de Salar. Mientras que en el caso del valle de SAC destacamos las concentraciones de material del Fondo de Cuenca. En dichos sectores detectamos, entre otros materiales, ejemplares de artefactos lanceolados unifaciales (Muscio 2004; López 2008). Los mismos serán considerados en el marco del segundo objetivo, orientado al estudio de la dispersión espacial de la tecnología de hojas, y que permitirán aumentar las muestras para la comparación.

\section{* Metodología}

Los pasos metodológicos planteados se estructuran según los dos objetivos presentados en la introducción de este trabajo. De acuerdo con el primer objetivo, centrado en la discusión de la aparición de la tecnología de hojas en la Puna Argentina, nos ocupamos de la identificación de artefactos vinculados con la misma. De este modo, realizamos una primera distinción en categorías generales, a saber: artefactos formatizados, desechos de talla y núcleos.

Para el caso de los artefactos formatizados consideramos las siguientes variables:

Módulo longitud/anchura. Consiste en el cociente entre las dos medidas. Los resultados iguales o mayores a dos corresponden a módulos laminares, mientras que los menores a dos corresponden a lascas (Aschero 1975). Fue medido sobre artefactos formatizados enteros.

Forma base. Se apuntó a identificar hojas, de acuerdo con los criterios presentados en la sección "aspectos generales sobre la tecnología de hojas".

Clase de roca. Esta distinción se realizó con la colaboración de personal de la Universidad de Buenos Aires especializado en geología (Licenciada Patricia Solá), mediante estudios petrográficos, así como con personal de la Universidad de Missouri, Estados Unidos, especializado en estudios de base físicoquímica para el caso de las obsidianas (Doctor Michael Glascock), empleando el método de espectroscopía por fluorescencia de rayos X (Mercuri y Restifo 2011).

Clase de artefacto formatizado. Siguiendo la propuesta de grupos tipológicos de Aschero (1975, 1983).

En relación a los desechos de talla, consideramos el módulo de longitud/anchura y la clase de roca, del mismo modo que para el caso de los artefactos formatizados. A su vez, agregamos la distinción por clase de desecho de talla siguiendo los criterios de Aschero (1975, 1983). Para esta distinción consideramos el "número mínimo de desechos" (NMD) (Aschero et al. 1993), basado en la sumatoria de los ejemplares enteros y aquellos fracturados con talón, con el fin de reducir el sobreconteo de desechos a causa de la fractura de los mismos.

En cuanto a los núcleos, las variables relevadas fueron las siguientes:

Negativos de extracción. Atendiendo a la relación entre las medidas de longitud y ancho, a fin de distinguir entre negativos de lascas y extracciones laminares, tomando en cuenta aquellas extracciones que se presentan como completas, correspondientes a los últimos lascados del núcleo.

Plataformas. Aquí atendimos a la detección de diferentes planos desde los cuales se efectuaron extracciones, observando también la dirección de las mismas.

Asimismo, al momento de presentar los datos sobre 
núcleos, en la sección de resultados, también incluimos otras evidencias relacionadas con la extracción de hojas, como es la presencia de una posible lasca de flanco de núcleo en el sitio Ramadas PN. La misma, si bien no es considerada un núcleo propiamente dicho, si posee un potencial de información que puede complementar a aquella obtenida a partir de los núcleos en sentido estricto, por este motivo su presentación acompaña a los datos relativos a dicha clase artefactual.

Por su parte, sobre la base del segundo objetivo planteado, orientado a la comparación de los artefactos lanceolados unifaciales de la cuenca de PG y el valle de SAC, en función de abordar la temática de circulación de información a través del espacio (Yacobaccio 1991; de Souza 2004; Franco et al. 2010), realizamos un análisis de base estadística. La expectativa general es que, a medida que aumentan los sesgos en la transmisión de información vinculada con la replicación de artefactos particulares, menor será la variación métrica que estos presentarán. En este caso, el sesgo en la transmisión cultural implica la replicación de artefactos siguiendo pautas e instrucciones específicas. Es decir, a mayor sesgo, mayor fidelidad de replicación, y en consecuencia, menor variación métrica. Esto puede ocurrir, por ejemplo, mediante mecanismos sociales como el aprendizaje por imitación (Boyd y Richerson 1985; Bettinger y Eerkens 1999). Sobre la base de esta expectativa medimos la longitud, ancho y espesor de cada pieza en milímetros. Posteriormente, calculamos el coeficiente de variación (CV) para cada una de ellas.

El CV representa una medida de la heterogeneidad de los valores de un conjunto de casos para una variable deter- minada (Collard et al. 2012). Su resultado puede variar de cero a uno y se puede expresar a modo de porcentajes. A medida que se aleja de cero, acercándose a uno, la heterogeneidad es cada vez mayor. Entonces, valores bajos de CV expresan baja variación, lo que sería coherente con la expectativa de mayores sesgos en la transmisión cultural (Cardillo 2002; López 2008). El coeficiente se calcula dividiendo el desvío estándar sobre la media, es decir:

$\mathrm{CV}=\mathrm{s} / \mathrm{m}$

Luego, con el fin de complementar la información obtenida a partir del CV, aplicamos el test estadístico denominado Mann-Withney, aplicable a conjuntos de datos que no se ajustan a distribuciones normales, tal como el caso que se analiza. El mismo permite medir la significancia de diferencias entre dos poblaciones estadísticas o conjuntos de datos supuestamente independientes (Hair et al. 2005). En nuestro caso, será útil para determinar si las diferencias en los valores medios de las dimensiones de longitud, ancho y espesor de los artefactos lanceolados unifaciales son significativas o no de acuerdo con el área de procedencia de las mismas, ya sea la cuenca de PG o el valle de SAC. Considerando la influencia de sesgos en la replicación de dichos artefactos, la expectativa es que las diferencias entre las dos muestras no sean significativas, correspondiendo ambas a una misma población estadística. Este procedimiento ha sido utilizado en diferentes estudios comparativos en la puna y otras regiones como Patagonia (Yacobaccio 1991; Franco et al. 2010). Todos los análisis estadísticos fueron realizados mediante el uso del programa Past 2.14 (Hammer et al. 2001), de uso gratuito.

\begin{tabular}{|l|c|c|c|c|c|}
\hline \multicolumn{1}{|c|}{ Sitio } & Artefactos Formalizados & Desechos de talla & Núcleos & Total n & Total \% \\
\hline Alero Cuevas (Capa F4) & 70 & 3675 & & 3745 & 73,8 \\
\hline Alero Cuevas (Capa F3) & 8 & 195 & & 203 & 4 \\
\hline Alero Cuevas (Capa F2) & 29 & 686 & 4 & 718 & 14 \\
\hline Ramadas PN (Capa C) & 8 & 366 & & 18 & 778 \\
\hline Colecc. Lan. Unif. PG & 18 & & & 11 & 0,5 \\
\hline Colecc. Lan. Unif. SAC & 11 & & 7 & 5073 & 100 \\
\hline Total n & 144 & 4922 & 0,1 & 100 & \\
\hline Total \% & 2,9 & 97 & & & \\
\hline
\end{tabular}

Tabla 2. Detalle de la muestra analizada. 


\section{* Identificación de hojas en Conjuntos del HOLOCENO TEMPRANO Y MEDIO}

Trabajamos sobre una muestra de 5073 artefactos, entre artefactos formatizados, núcleos y desechos de talla, cuyo detalle se presenta en la Tabla 2.

\section{Sitio Alero Cuevas}

Artefactos formatizados. Comenzamos por la observación de la tendencia de los valores de módulo longitud/anchura. En este caso hicimos la excepción de centrarnos en las capas $\mathrm{F}_{4}(\mathrm{n}=33)$ y $\mathrm{F}_{2}(\mathrm{n}=12)$, dado que la capa $\mathrm{F}_{3}$ presenta una muestra muy pequeña de piezas enteras como para aproximar tendencias $(n=3)$. En la Figura 2 observamos que la capa F2 presenta una distribución de mayor amplitud en relación a $\mathrm{F}_{4}$, es decir, un mayor desvío estándar, lo que implica una mayor diversidad de valores. A su vez, observamos que la media de los valores es mayor para el caso de la capa F2, ubicándose por encima del valor de $2 \mathrm{~mm}$ (línea horizontal gruesa), lo que indica, a su vez, una tendencia más marcada hacia los módulos laminares en dicha capa. Por su parte, el análisis individual de los ejemplares de $\mathrm{F}_{3}$ indica la presencia de dos casos de módulo de lasca y uno laminar.

Al observar los datos de clases de formas base, se destaca el predominio de las lascas en $\mathrm{F}_{4}$, superando el 70\%,

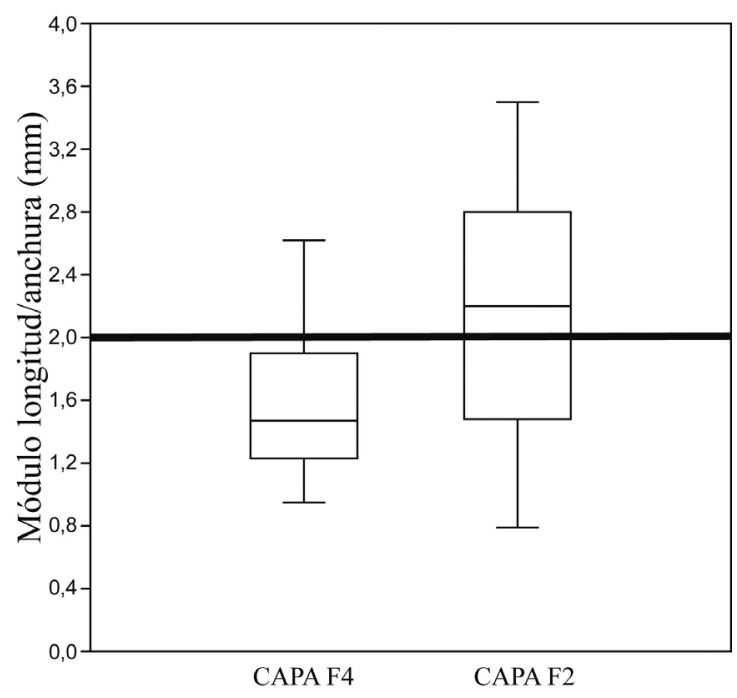

Figura 2. Distribuciones de valores del módulo longitud anchura para artefactos formatizados del sitio Alero Cuevas. mientras que en $\mathrm{F}_{3}$ el predominio es absoluto. Por su parte, en la capa $\mathrm{F}_{2}$ la frecuencia relativa de hojas aumenta al punto de igualarse con las de lascas, con frecuencias que rondan el $40 \%$ cada una (Tablas 3 y 4 ). En relación a las rocas (Tabla 3 ), se destaca el aumento de las frecuencias de andesita, pasando de un 14\% en $\mathrm{F}_{4}$ a un $37 \%$ en $\mathrm{F}_{2}$. Este aumento se asocia con el incremento de hojas. Es decir, los artefactos formatizados sobre hojas son a su vez mayormente tallados en andesita. Dicha roca corresponde al orden de lo local (López 2008), ubicándose a unos $10 \mathrm{~km}$ del sitio Alero Cuevas. Por su parte, en la capa F4 predomina la obsidiana de Quirón, roca considerada no local, ubicada a unos $40 \mathrm{~km}$ del sitio. En resumen, destacamos que el patrón de aumento de hojas se asocia a un patrón de aumento de roca local. Por su parte, la capa $\mathrm{F}_{3}$ presenta el predomino de obsidiana de Quirón también, aunque los datos deben tomarse con precaución, dado el pequeño tamaño de la muestra. Una mención especial es para el caso de las obsidianas lejanas. En la capa F4 destacamos la presencia de obsidiana de Archibarca (ubicada a unos $150 \mathrm{~km}$ al sur), Zapaleri (200 km hacia el norte), y un caso de procedencia desconocida (color gris), pero que también ha sido detectada en contextos arqueológicos del norte de Chile (Mercuri y Restifo 2011). Asimismo, la obsidiana desconocida reaparece en $\mathrm{F}_{3}$, mientras que la obsidiana de Archibarca reaparece en F2.

Al considerar la forma base en relación a la clase de artefacto formatizado (Tabla 4), observamos, especialmente en F2, que tanto hojas como lascas son utilizadas para la confección de una variedad de artefactos de filo retocado, incluyendo piezas con uno o más filos. En este caso, de los 12 artefactos formatizados sobre hojas, 11 de ellos son asignables a la categoría general lanceolada unifacial, que engloba a las conocidas como raedera, cuchillo, raspador, etc. Se trata de piezas de limbo lanceolado, sección planoconvexa, ángulos de filo variables, y un patrón de lascados en cara ventral, reduciendo el espesor del bulbo (Figura 3). Considerando la capa $\mathrm{F}_{4}$, lo que observamos es una diversidad de artefactos formatizados sobre lascas. Dicha diversidad persiste en la capa F2, pero con la diferencia de la presencia de hojas como forma base también recurrente. En relación a las demás clases artefactuales, destacamos la presencia de artefactos diagnósticos tales como las puntas de proyectil triangulares apedunculadas en $\mathrm{F}_{4}$, así como lanceoladas en $\mathrm{F}_{3}$, y lanceoladas de tamaño más pequeño en F2. Remitimos a las publicacio- 


\begin{tabular}{|c|c|c|c|c|c|c|c|}
\hline Sitio & Roca & Lasca & Hoja & Guijarro & No dif. & Total $\mathbf{n}$ & Total \% \\
\hline \multirow{5}{*}{ Alero Cuevas Capa F4 } & OQ & 34 & 1 & & 12 & 47 & 67,14 \\
\hline & OBS LEJ. & 3 & & & & 3 & 4,3 \\
\hline & AN & 8 & 1 & & 1 & 10 & 14,36 \\
\hline & SIL & 4 & & & 1 & 5 & 7,1 \\
\hline & Otras & 3 & 1 & 1 & & 5 & 7,1 \\
\hline \multirow{2}{*}{ Totales } & Total $n$ & 52 & 3 & 1 & 14 & 70 & 100 \\
\hline & Total \% & 74,3 & 4,3 & 1,4 & 20 & 100 & \\
\hline \multirow{5}{*}{ Alero Cuevas Capa F3 } & OQ & 1 & & & 2 & 3 & 37,5 \\
\hline & OBS LEJ. & 1 & & & & 1 & 12,5 \\
\hline & AN & 1 & & & 1 & 2 & 25 \\
\hline & CTA & & & & 1 & 1 & 12,5 \\
\hline & SIL & & & & 1 & 1 & 12,5 \\
\hline \multirow{2}{*}{ Totales } & Total n & 3 & & & 5 & 8 & 100 \\
\hline & Total \% & 37,5 & & & 62,5 & 100 & \\
\hline \multirow{4}{*}{ Alero Cuevas Capa F2 } & OQ & 7 & & & 2 & 9 & 24,3 \\
\hline & OBS LEJ. & & 1 & & & 1 & 2,7 \\
\hline & AN & 4 & 9 & & 1 & 14 & 37,8 \\
\hline & Otras & 1 & 2 & & 2 & 5 & 13,5 \\
\hline \multirow{2}{*}{ Totales } & Total $n$ & 12 & 12 & & 5 & 29 & 100 \\
\hline & Total \% & 41,4 & 41,4 & & 17,2 & 100 & \\
\hline
\end{tabular}

Tabla 3. Formas base y rocas correspondientes al sitio Alero Cuevas. Referencias: OQ: Obsidiana de Quirón; OBS LEJ: Obsidianas lejanas; AN: Andesita; SIL: Silícea; y CTA: Cuarcita.

nes correspondientes para más detalle (Restifo y Hoguin 2012; Restifo 2013).

Desechos de talla. Comenzando por los valores de módulo longitud/anchura (Figura 4), lo que se observa es un patrón levemente decreciente hacia F2, a juzgar por la media registrada en los conjuntos de cada capa. El caso de $\mathrm{F}_{4}$ es el que presenta la mayor amplitud de valores, no sólo por su distribución sino también por la presencia de outliers que representan casos de piezas notablemente elongadas. La capa F3 presenta la distribución de menor amplitud, mientras que F2 se encuentra en una posición intermedia en relación a la distribución de sus valores. Asimismo, la media para el caso de las tres capas se ubica por debajo del límite de módulos laminares.

En relación a las rocas (Tabla 5), observamos el predominio de la roca no local, representada principalmente por la obsidiana de Quirón, en las tres capas. Sin embargo, en el caso de F2 la diferencia entre obsidiana de Quirón y andesita - roca local — es menor, mostrando el aumento de frecuencias de esta última.

Por su parte, la información de clases de desechos de talla (Tabla 5) indica el predominio claro de lascas en las tres capas, y dentro de esta categoría, la predominante es la lasca angular. A su vez, observamos un leve aumento en la representación de las lascas de arista simple y doble a través de las tres capas, con las frecuencias mayores para el caso de F2 (Figura 5). Asimismo, para el caso de esta capa las lascas de arista simple y doble aparecen mayormente representadas en andesita, roca en la que destacamos la formatización de artefactos lanceolados unifaciales también, mientras que para el caso de $\mathrm{F}_{4} \mathrm{y}$ $\mathrm{F}_{3}$ dichas clases de desecho de talla corresponden a rocas diferentes, sin predominancia clara de ninguna. A su 

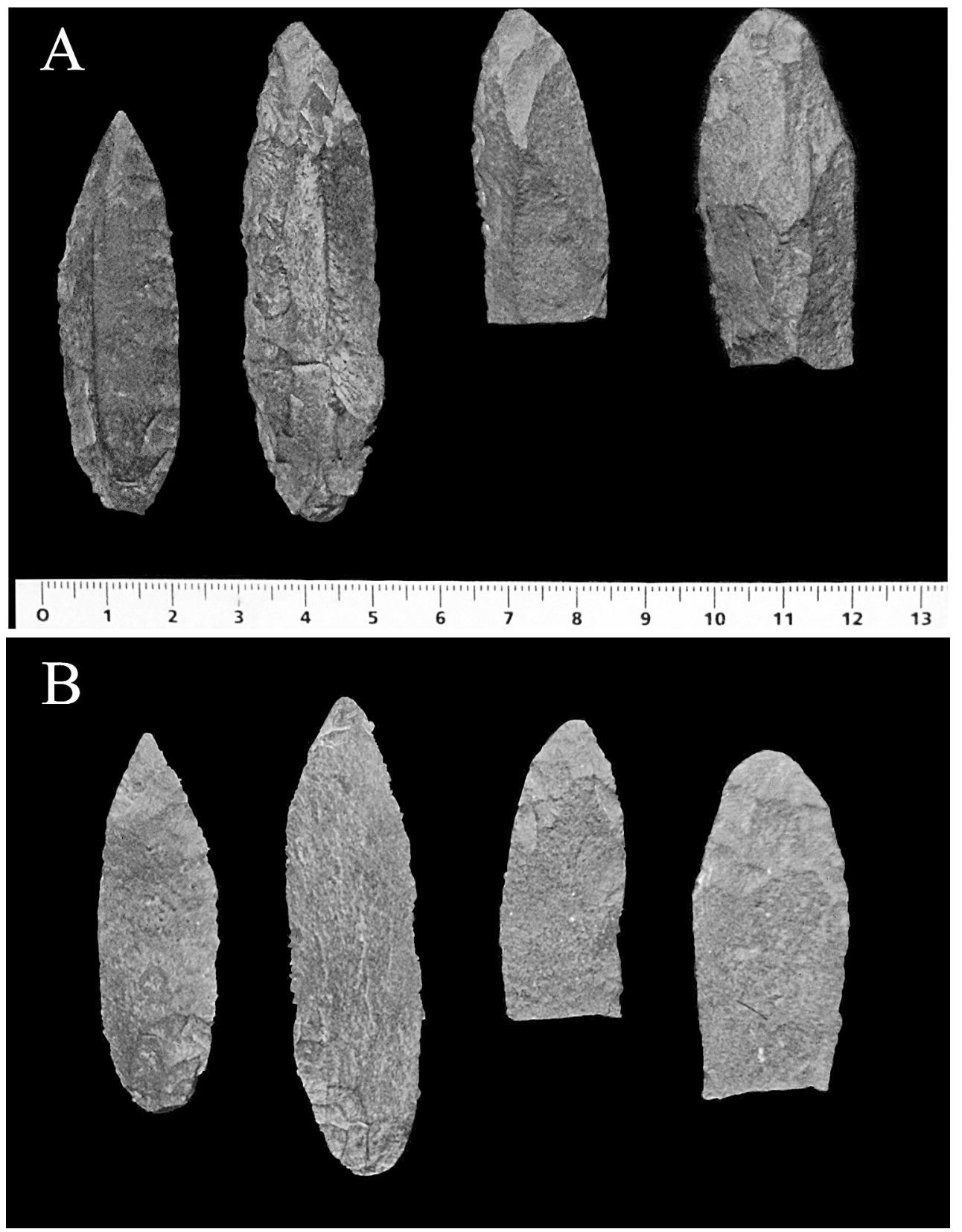

Figura 3. Ejemplares de artefactos lanceolados unifaciales provenientes del sitio Alero Cuevas. A) Artefactos vistos en cara dorsal. B) Artefactos vistos en cara ventral, nótese los patrones de lascado, que reducen el espesor del bulbo (parte inferior en los dos primeros, parte superior en los dos restantes).

vez, los desechos clasificables como hojas se presentan en valores muy bajos, e incluso ausentes, como en el caso de la capa $\mathrm{F}_{3}$.

Tomando en cuenta las obsidianas lejanas, destacamos la presencia de la obsidiana de Zapaleri $(200 \mathrm{~km}$ hacia el norte) y Archibarca ( $150 \mathrm{~km}$ hacia el sur) para el caso de F4, la obsidiana de Archibarca para el caso de F3, y Archibarca y Zapaleri para el caso de F2.

Núcleos. Hasta el momento hemos recuperado un único núcleo en las excavaciones del sitio Alero Cuevas (Figu- 


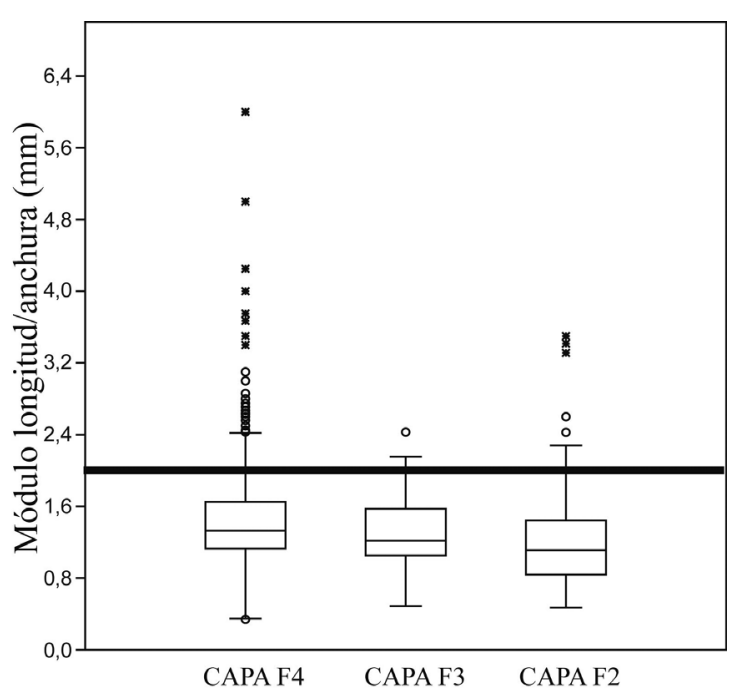

Figura 4. Distribuciones de valores del módulo longitud/anchura para desechos de talla del sitio Alero Cuevas.

ra 6). El mismo es de relevancia ya que fue hallado en la capa F2, asociado a artefactos lanceolados unifaciales. Su materia prima es andesita, lo que es coherente con la roca de los artefactos formatizados sobre hoja hallados. El ejemplar presenta remanente de corteza y una morfología general prismática. A su vez, es clara la presencia de dos plataformas opuestas, a partir de las cuales se observan diferentes negativos de extracciones, también en direcciones opuestas, predominando aquellas de módulo laminar. En los bordes de ambas plataformas son visibles rasgos de abradido y pequeños negativos de lascado, pro- bablemente relacionados con la preparación del frente de extracción de formas base.

\section{Ramadas Perfil Norte}

Artefactos formatizados. Dado que la muestra de piezas enteras presenta pocos ejemplares para estimar tendencias $(n=3)$, no presentamos gráficos de cajas de módulo longitud/anchura, como en la sección de Alero Cuevas. De todas formas, el examen individual de las piezas $(n=8)$, permitió la identificación de siete casos correspondientes a módulos laminares, mostrando el predominio de los mismos. Si bien la muestra es pequeña, mantiene coherencia con lo observado en la capa F2, ubicada temporalmente también en la porción final del Holoceno Medio. Asimismo, estas siete piezas con módulo laminar están formatizadas sobre hojas, mostrando el predominio de dicha forma base en el conjunto (Tablas 6 y 7 ).

En cuanto a las rocas (Tabla 6), identificamos roca metamórfica gris y cuarcita. Las mismas corresponden al orden de lo local, ubicándose a una distancia promedio de $5 \mathrm{~km}$ respecto del sitio. A su vez, tomando en cuenta las clases de artefactos formatizados (Tabla 7), observamos la presencia de artefactos formatizados con variedad de filos, sobre forma base de hojas. De los siete artefactos formatizados sobre hojas, seis de ellos fueron clasificados como lanceolados unifaciales.

Desechos de talla. Tomando en cuenta los valores de mó-

\begin{tabular}{|c|c|c|c|c|c|c|c|c|c|c|c|c|c|c|c|c|c|}
\hline Sitio & F. base & $\mathrm{Rp}$ & $\mathrm{Rd}$ & CFR & Perf. & $\mathrm{Ph}$ & $\mathrm{Pp}$ & Pref. Pp & $\mathrm{Rd}+\mathrm{CFR}+\mathrm{Ph}$ & $\mathrm{Rd}+\mathrm{Rd}+\mathrm{Ph}$ & $\mathrm{Rd}+\mathrm{Rp}+\mathrm{Ph}$ & $\mathrm{Rp}+\mathrm{CFR}$ & ADFS & FNDAF & Biface & Total $\mathrm{n}$ & Total \% \\
\hline \multirow{4}{*}{$\begin{array}{c}\text { Alero } \\
\text { Cuevas } \\
\text { Capa F4 }\end{array}$} & Lasca & 4 & 3 & 4 & 1 & & 6 & 1 & 1 & 1 & 3 & 1 & 18 & 9 & & 52 & 74,3 \\
\hline & Hoja & 1 & & 2 & & & & & & & & & & & & 3 & 4,3 \\
\hline & Guijarro & & & & & & & & & & & & 1 & & & 1 & 1,4 \\
\hline & No dif. & & & & & & 5 & & & & 1 & & & 8 & & 14 & 20 \\
\hline \multirow{2}{*}{ Totales } & Total $\mathrm{n}$ & 5 & 3 & 6 & 1 & & 11 & 1 & 1 & 1 & 4 & 1 & 19 & 17 & & 70 & 100 \\
\hline & Total \% & 7,1 & 4,3 & 8,6 & 1,4 & & 15,8 & 1,4 & 1,4 & 1,4 & 5,7 & 1,4 & 27,2 & 24,3 & & 100 & \\
\hline \multirow{3}{*}{$\begin{array}{c}\text { Alero } \\
\text { Cuevas } \\
\text { Capa F }\end{array}$} & Lasca & & & 1 & & & 1 & 1 & & & & & & & & 3 & 37,5 \\
\hline & Hoja & & & & & & & & & & & & & & & & \\
\hline & No dif. & & & & & & 3 & 1 & & & & & & 1 & & 5 & 62,5 \\
\hline \multirow{2}{*}{ Totales } & Total $\mathrm{n}$ & & & 1 & & & 4 & 2 & & & & & & 1 & & 8 & 100 \\
\hline & Total \% & & & 12,5 & & & 50 & 25 & & & & & & 12,5 & & 100 & \\
\hline \multirow{3}{*}{$\begin{array}{c}\text { Alero } \\
\text { Cuevas } \\
\text { Capa F2 }\end{array}$} & Lasca & 1 & 3 & & & 1 & 1 & & & & & & 2 & 2 & 2 & 12 & 41,4 \\
\hline & Hoja & & 6 & 1 & & 2 & & & & 2 & & & 1 & & & 12 & 41,4 \\
\hline & No dif. & & & & & & & & & & & & & 5 & & 5 & 17,2 \\
\hline \multirow{2}{*}{ Totales } & Total $\mathrm{n}$ & 1 & 9 & 1 & & 3 & 1 & & & 2 & & & 3 & 7 & 2 & 29 & 100 \\
\hline & Total \% & 3,5 & 30,2 & 3,5 & & 10,3 & 3,5 & & & 7 & & & 10,5 & 24,5 & 7 & 100 & \\
\hline
\end{tabular}

Tabla 4. Formas base y clases de artefactos formatizados correspondientes al sitio Alero Cuevas. Referencias: Rp: Raspador; Rd: Raedera; CFR; Cuchillo de filo retocado; Perf: Perforador; Pf: Punta herramienta; Pref. Pp: Preforma de punta de proyectil; Pp: Punta de proyectil; ADFS: Artefacto de formatización sumaria; y FNDAF: Fragmento no diferenciado de artefacto formatizado. 


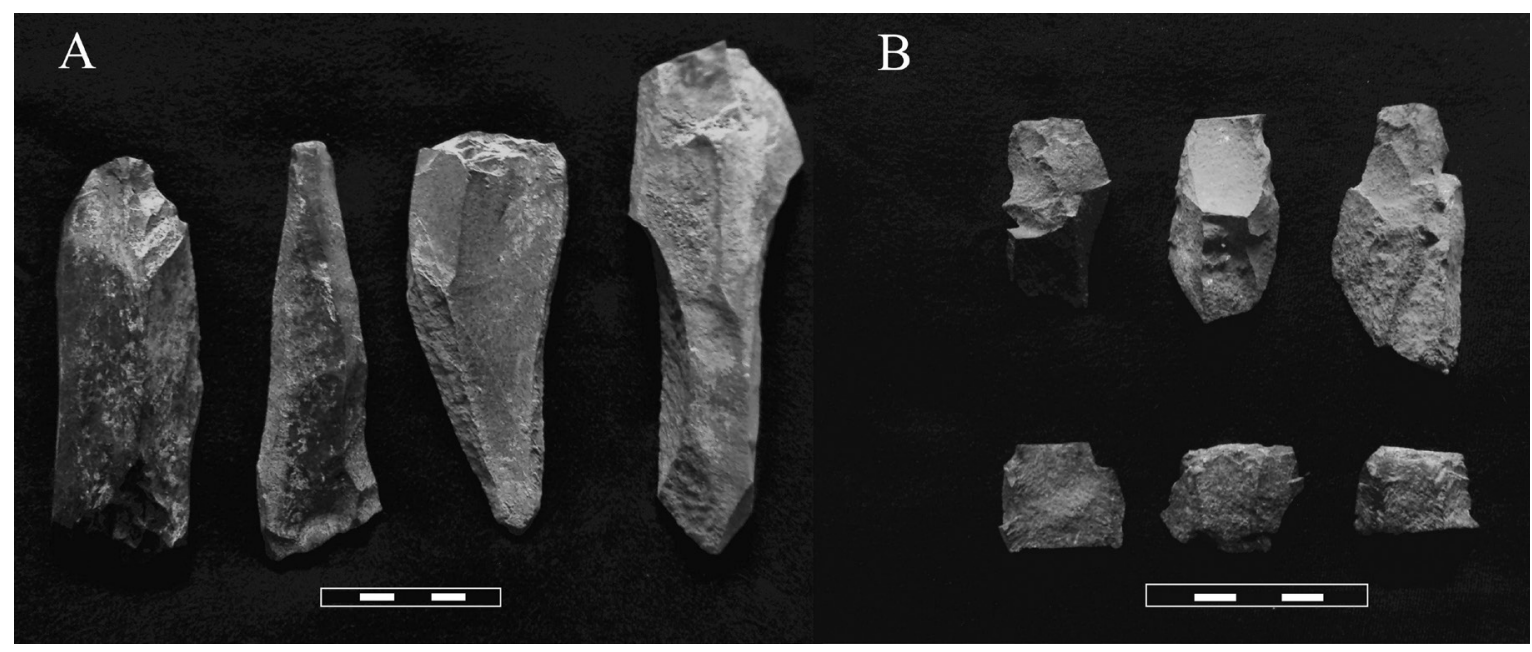

Figura 5. Ejemplares de desechos de talla vinculados con la tecnología de hojas (preparación de núcleos). A) Hojas no modificadas provenientes de Ramadas PN, nótese el ejemplar de la derecha, correspondiente a una hoja sobrepasada. B) Desechos de talla provenientes de la capa F2 del sitio Alero Cuevas.

\begin{tabular}{|c|c|c|c|c|c|c|c|c|c|c|c|}
\hline \multirow[b]{2}{*}{ Sitio } & \multirow[b]{2}{*}{ Roca } & \multicolumn{5}{|c|}{ Lasca } & \multicolumn{2}{|c|}{ Hoja } & \multirow[b]{2}{*}{ No dif. } & \multirow[b]{2}{*}{ Total $\mathbf{n}$} & \multirow[b]{2}{*}{ Total \% } \\
\hline & & C/corteza & Plana & A. simple & A. doble & Angular & A. simple & A. doble & & & \\
\hline \multirow{5}{*}{$\begin{array}{c}\text { Alero Cuevas } \\
\text { Capa } F_{4}\end{array}$} & OQ & 12 & 8 & 24 & 2 & 725 & 4 & 1 & 124 & 900 & 63 \\
\hline & OBS LEJ. & 3 & 2 & 4 & & 210 & o & & 37 & 256 & 11,4 \\
\hline & AN & & 31 & 12 & & 245 & $\circ$ & & 25 & 313 & 16,5 \\
\hline & SIL & & & 1 & & 23 & & & 3 & 27 & 1,3 \\
\hline & Otras & 1 & 5 & 3 & 1 & 65 & 0 & & 22 & 97 & 7,8 \\
\hline \multirow{2}{*}{ Totales } & Total $\mathbf{n}$ & 16 & 46 & 44 & 3 & 1268 & 4 & 1 & 211 & 1593 & 100 \\
\hline & Total \% & 1,4 & 4,2 & 4 & 0,3 & 70,3 & 0,7 & 0,1 & 19 & 100 & \\
\hline \multirow{4}{*}{$\begin{array}{c}\text { Alero Cuevas } \\
\text { Capa F } 3\end{array}$} & $\mathrm{OQ}$ & 2 & 4 & 2 & 2 & 53 & & & 6 & 69 & 65,1 \\
\hline & OBS LEJ. & & & & & 2 & & & & 2 & 1,9 \\
\hline & AN & & 2 & 2 & & 17 & & & 4 & 25 & 23,6 \\
\hline & Otras & & 2 & 1 & & 6 & & & 1 & 10 & 9,4 \\
\hline \multirow{2}{*}{ Totales } & Total $\mathbf{n}$ & 2 & 8 & 5 & 2 & 78 & & & 11 & 106 & 100 \\
\hline & Total \% & 1,9 & 7,6 & 4,7 & 1,9 & 73,6 & & & 10,3 & 100 & \\
\hline \multirow{4}{*}{$\begin{array}{c}\text { Alero Cuevas } \\
\text { Capa F2 }\end{array}$} & OQ & 18 & 3 & 5 & 3 & 147 & & & 17 & 193 & 47,6 \\
\hline & OBS LEJ. & 4 & 1 & & & & & & & 5 & 1,2 \\
\hline & AN & 3 & 27 & 18 & 11 & 84 & & & 11 & 154 & 38 \\
\hline & Otras & 3 & 11 & 3 & & 32 & 1 & & 3 & 53 & 13,2 \\
\hline \multirow{2}{*}{ Totales } & Total n & 28 & 42 & 26 & 14 & 263 & 1 & & 31 & 405 & 100 \\
\hline & Total \% & 6,9 & 10,4 & 6,4 & 3,5 & 65 & 0,2 & & 7,6 & 100 & \\
\hline
\end{tabular}

Tabla 5. Clases de desechos de talla y rocas correspondientes al sitio Alero Cuevas. Referencias: A. simple: arista simple; A. doble: arista doble.

dulo longitud/anchura (Figura 7), se observa una media inferior al límite de módulos laminares. A su vez, observamos una distribución amplia, que contempla valores correspondientes a dichos módulos en su tendencia de dispersión, aunque la tendencia central se incline hacia módulos de menor valor. En relación a las clases de desechos de talla, en la Tabla 8 se destaca el predominio de las lascas, y dentro de esta categoría observamos el predominio de las lascas angulares. Por su parte, las lascas de arista simple y doble aparecen en bajas frecuencias, del 


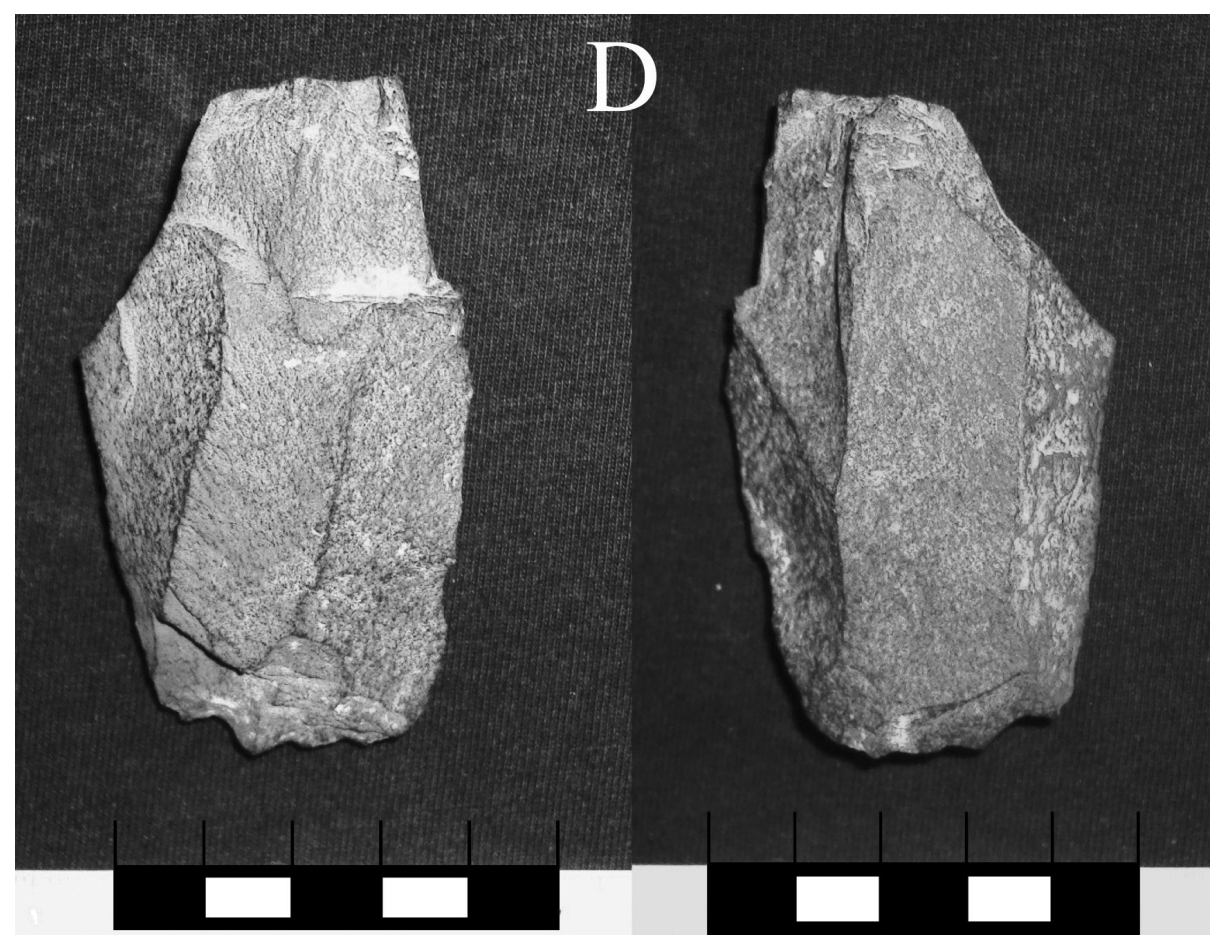

Figura 6. Núcleo recuperado en el sitio Alero Cuevas. Ver texto para su descripción.

\begin{tabular}{|c|c|c|c|c|c|c|}
\hline Sitio & Roca & Lasca & Hoja & No dif. & Total $\mathbf{n}$ & Total \% \\
\hline \multirow{2}{*}{ Ramadas PN Capa C } & MFG & 3 & 4 & & 7 & 87,5 \\
\cline { 2 - 8 } & CTA & 1 & & & 1 & 12,5 \\
\hline \multirow{2}{*}{ Totales } & Total $\mathbf{n}$ & 4 & 4 & & 8 & 100 \\
\cline { 2 - 8 } & Total \% & 50 & 50 & & 100 & \\
\hline
\end{tabular}

Tabla 6. Formas base y rocas correspondientes al sitio Ramadas PN. Referencias: MFG: Roca metamórfica gris.

\begin{tabular}{|c|c|c|c|c|c|c|c|}
\hline Sitio & F.base & Rd & Rd + Rd & CFR+Ph & FNDAF & Total n & Total \% \\
\hline \multirow{4}{*}{ Ramadas PN Capa C } & Lasca & 3 & & & 1 & 4 & 50 \\
\cline { 2 - 8 } & Hoja & 2 & 1 & 1 & & 4 & 50 \\
\cline { 2 - 9 } & No dif. & & & & & & \\
\hline \multirow{2}{*}{ Totales } & Total n & 5 & 1 & 1 & 1 & 8 & 100 \\
\cline { 2 - 8 } & Total \% & 62,5 & 12,5 & 12,5 & 12,5 & 100 & \\
\hline
\end{tabular}

Tabla 7. Formas base y clases de artefactos formatizados correspondientes al sitio Ramadas PN.

mismo modo que las demás categorías. Si bien las hojas aparecen en bajas frecuencias, puede observarse su mayor representación en roca metamórfica gris, que es, a su vez, la roca en la que están tallados la mayor parte de los artefactos formatizados sobre hojas, y por otra parte, la roca predominante en el conjunto. Asimismo, entre las hojas destacamos la presencia de ejemplares de gran tamaño (Figura 5), los que constituyen formas base potenciales.
Núcleos y otras evidencias de extracción de hojas. Comenzando por el ejemplar A (Figura 8-A), destacamos que en él identificamos tres plataformas. Dos de ellas se ubican en los extremos. Desde ellas parten diferentes extracciones, entre las que se destacan aquellas asignables a extracciones laminares como lasca. A su vez, la tercera plataforma se ubica en el sector medio, y a partir de ella se observan negativos correspondientes a lascas. Se trata de un nú- 


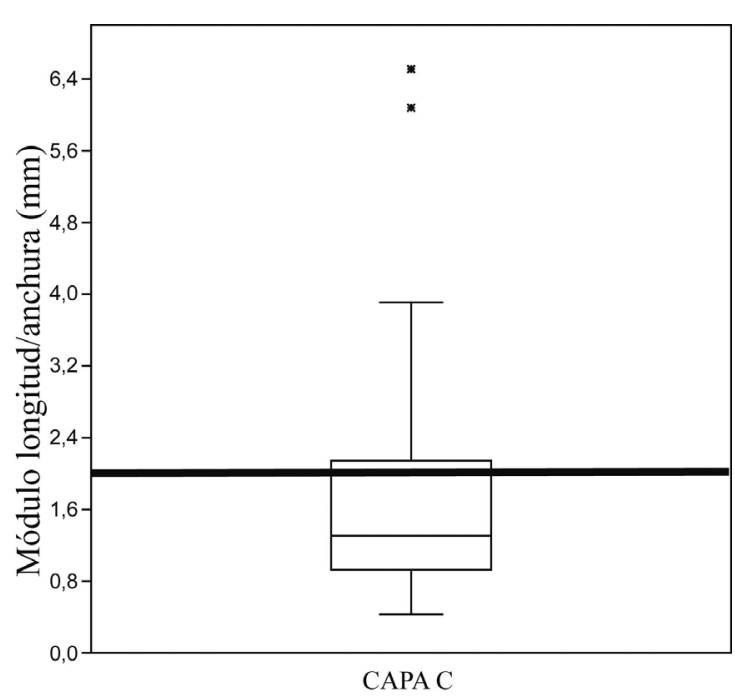

Figura 7. Distribuciones de valores del módulo longitud/anchura para desechos de talla del sitio Ramadas PN.

cleo que pudo haber cumplido la doble función de proporcionar tanto lascas como extracciones laminares.

Por su parte, el ejemplar B (Figura 8-B) presenta un remanente de corteza que cubre casi la totalidad de una de las caras. Se destaca su reducido espesor, con un valor de $18 \mathrm{~mm}$, lo que permite suponer que se encontraría agotado. En este núcleo identificamos dos plataformas, ubicadas en sus dos extremos. Especialmente a partir de una de ellas es que se observan negativos asignables a extracciones laminares. Estas cubren casi por completo la cara trabajada. A su vez, una de las plataformas presenta rasgos de abrasión en su borde.

La pieza restante recuperada en Ramadas PN, ejemplar $\mathrm{C}$, no es un núcleo propiamente dicho sino una lasca. Sin embargo, ella misma evidencia la extracción de formas base laminares en el sitio, dados sus negativos de extracción y remanente de una plataforma, por eso su inclusión en la presente sección. Su eje técnico se ubica en dirección subparalela a la parte visible de la plataforma. Siguiendo a Aschero (1975, 1983), este fragmento puede clasificarse como "lasca de flanco de núcleo". El remanente de plataforma presenta rasgos de abrasión, al igual que el caso anterior. A partir de dicha plataforma se observan dos negativos de extracciones laminares que cubren de manera casi completa la cara dorsal.

\section{* comparación de los artefactos lanceola-} DOS UNIFACIALES DE LA CUENCA DE PG Y EL VALLE DE SAC

Iniciamos esta sección recordando que las muestras de artefactos lanceolados unifaciales empleadas fueron recuperadas en los sitios estratificados tratados hasta aquí,

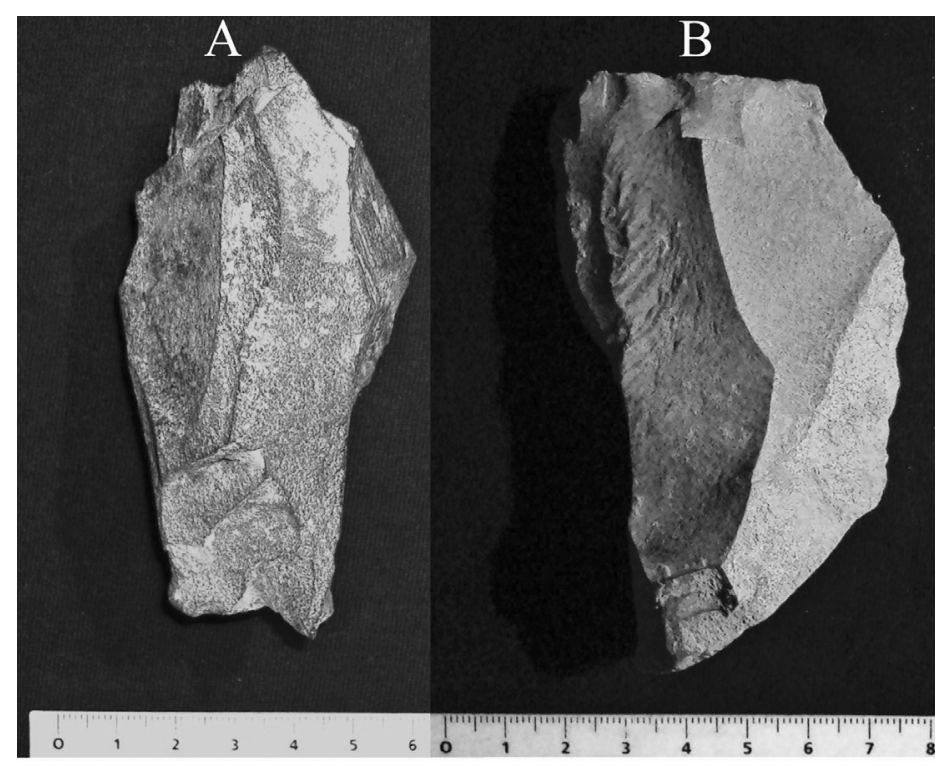

Figura 8. A) y B) Núcleos recuperados en el sitio Ramadas PN. 


\begin{tabular}{|c|c|c|c|c|c|c|c|c|c|c|c|}
\hline \multirow[b]{2}{*}{ Sitio } & \multirow[b]{2}{*}{ Roca } & \multicolumn{5}{|c|}{ Lasca } & \multicolumn{2}{|c|}{ Hoja } & \multirow[b]{2}{*}{ No dif. } & \multirow[b]{2}{*}{ Total n } & \multirow[b]{2}{*}{ Total \% } \\
\hline & & $\mathrm{C} /$ corteza & Plana & A. simple & A. doble & Angular & A. simple & A. doble & & & \\
\hline \multirow{3}{*}{$\begin{array}{l}\text { Ramadas } \\
\text { PN Capa C }\end{array}$} & MFG & 2 & 22 & 4 & 8 & 73 & 2 & 8 & 1 & 120 & 56,3 \\
\hline & CTA & 2 & 14 & 5 & 1 & 46 & & 1 & 7 & 76 & 35,7 \\
\hline & Otras & & 2 & & & 12 & & & 3 & 17 & 8 \\
\hline \multirow{2}{*}{ Totales } & Total n & 4 & 38 & 9 & 9 & 131 & 2 & 9 & 11 & 213 & 100 \\
\hline & Total \% & 1,9 & 17,8 & 4,2 & 4,2 & 61,5 & 0,9 & 4,2 & 5,3 & 100 & \\
\hline
\end{tabular}

Tabla 8. Clases de desechos de talla y rocas correspondientes al sitio Ramadas PN.

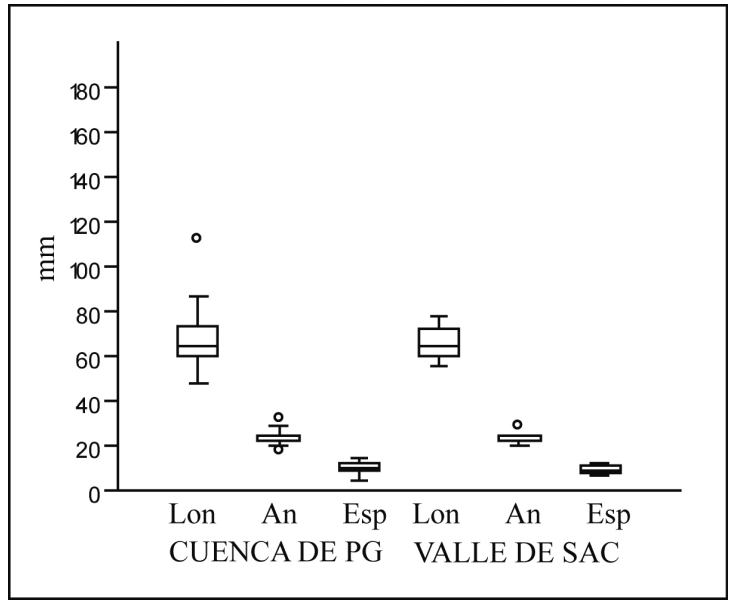

Figura 9. Distribuciones de datos de longitud, ancho y espesor de las muestras de artefactos lanceolados unifaciales de la cuenca de PG y el valle de SAC. Referencias: Lon: longitud; An: ancho; Esp: espesor.

Alero Cuevas y Ramadas PN, así como en sitios arqueológicos de superficie de las dos áreas de estudio en cuestión, cuenca de PG y valle de SAC. La muestra del sitio Alero Cuevas es de 11 piezas, a las que sumamos otras 18 provenientes de recolecciones de superficie en el borde de Salar $(n=11)$ y en Quebradas $(n=7)$. Para el caso del valle de SAC, a las seis piezas obtenidas en el sitio Ramadas
PN, sumamos otras 11 obtenidas en recolecciones de superficie en el Fondo de Cuenca.

En primer lugar, presentamos los datos de estadística descriptiva y coeficiente de variación para las dimensiones de longitud, ancho y espesor (Tabla 9, Figura 9). Tanto la muestra del valle de SAC como la muestra de la cuenca de PG presentan baja variación en sus atributos métricos, ubicándose todas las dimensiones por debajo del 24\%, según los valores de coeficiente de variación. Esto, a su vez, sugiere estandarización en esta clase artefactual.

En segundo lugar, destacamos que en este marco de baja variación, la muestra del valle de SAC posee rangos de variación menores, en comparación con la muestra de la cuenca de PG. No obstante, si bien encontramos diferencias en las variables métricas de ambas muestras, las mismas no se diferencian en términos estadísticos. Esto lo corroboramos a partir de la aplicación del test estadístico denominado Mann-Whitney. La aplicación del test dio como resultado que no existen diferencias significativas para ningún par de variables, con los siguientes valores: Longitud ( $\mathrm{T}=\mathrm{Ub}$ : 179,5; $\mathrm{p}=1$ ), ancho ( $\mathrm{T}=\mathrm{Ub}$ : 198; $\mathrm{p}=$ $0,2747$ ) y espesor ( $T=U b: 170 ; p=0,08)$.

\begin{tabular}{|c|l|c|c|c|c|c|c|c|}
\hline \multicolumn{1}{|c|}{ ÁREA } & \multicolumn{1}{|c|}{ Variable } & $\mathbf{n}$ & Media & DS & Min. & Max. & Varianza & CV \\
\hline \multirow{4}{*}{ Cuenca de PG } & Longitud & 24 & 67,47 & 13,99 & 48 & 112,5 & 195,78 & 0,2 \\
\cline { 2 - 11 } & Ancho & 29 & 23,53 & 3,32 & 17,4 & 32,7 & 11,04 & 0,14 \\
\cline { 2 - 11 } & Espesor & 29 & 10,37 & 2,52 & 5 & 14,8 & 6,36 & 0,24 \\
\hline \multirow{3}{*}{ Valle de SAC } & Longitud & 15 & 65,92 & 6,33 & 56 & 77,3 & 40,12 & 0,09 \\
\cline { 2 - 11 } & Ancho & 17 & 24,01 & 2,33 & 20,4 & 29 & 5,44 & 0,09 \\
\cline { 2 - 10 } & Espesor & 17 & 9,19 & 1,55 & 7 & 12 & 2,41 & 0,16 \\
\hline
\end{tabular}

Tabla 9. Estadística descriptiva para las muestras de artefactos lanceolados unifaciales de la cuenca de PG y el valle de SAC (recuperados en capa y en superficie). Los valores de "n" varían según si fue o no posible realizar la medición para el caso de algunos ejemplares fragmentados. 
Tomando en cuenta estos resultados podemos decir que, a pesar de las diferencias métricas registradas, las muestras consideradas no presentan diferencias estadísticamente significativas según el área de la cual provienen. Esto implica que pertenecerían a una misma población estadística, lo que puede relacionarse con procesos de transmisión cultural en la escala espacial, implicando al menos la circulación de información en espacios amplios, lo que discutiremos en la próxima sección.

\section{* Discusión}

La presente sección se estructura sobre la base de los objetivos planteados; el primero de ellos centrado en la discusión de la aparición de la tecnología de hojas en la Puna de Salta, mientras que el segundo está centrado en el abordaje de su dispersión espacial en dicha región. Respecto del primer objetivo, una serie de indicadores nos permiten sostener que la señal arqueológica más clara de tecnología de hojas ocurre hacia ca. $5200 \mathrm{AP}$, y se prolonga al menos hasta los ca. $4200 \mathrm{AP}$, según los fechados obtenidos en las capas F2 y C de los sitios Alero Cuevas y Ramadas PN (Tabla 2). De acuerdo con este planteamiento, un primer indicio está dado por la tendencia hacia módulos laminares en artefactos formatizados, en la capa F2 de Alero Cuevas, así como el predominio de artefactos con dicho módulo en el sitio Ramadas PN. Este primer indicio se confirma luego con el aumento de los artefactos formatizados sobre hojas, en la misma capa de Alero Cuevas, alcanzando la paridad con aquellos formatizados sobre lascas. Del mismo modo, las hojas son predominantes como forma base de los artefactos formatizados del sitio Ramadas PN. Esto marca una diferencia con los contextos del Holoceno Temprano e inicios del Holoceno Medio, en los que las formas base de hojas aparecen como ejemplares aislados o directamente ausentes, predominando las lascas.

Asimismo, decir que una pieza es "clasificable" como hoja implica resaltar ciertos atributos de la misma, especialmente en su cara dorsal. Sin embargo, para hablar de un sistema tecnológico propiamente dicho, consideramos que es necesario documentar la presencia de otra clase de piezas asociadas, como los núcleos orientados a tal fin, lo que daría cuenta de un comportamiento pautado, dirigido a la obtención de dicha clase de productos. Este es el caso, nuevamente, de los contextos de finales del Holoceno Medio en la Puna de Salta. En este sentido, en las capas F2 y C de Alero Cuevas y Ramadas PN, respectivamente, se destaca la presencia de núcleos con evidencia de extracciones laminares asociados a artefactos formatizados sobre hojas. Asimismo, la evidencia de desechos de talla acompaña este patrón. De este modo, en Alero Cuevas observamos una tendencia, si bien leve, hacia el aumento de las lascas de arista simple y doble hacia fines del Holoceno Medio, las que pueden constituir desechos de talla de un sistema tecnológico orientado a la producción de hojas. Por su parte, en Ramadas PN recuperamos hojas no modificadas cuyo tamaño sugiere la presencia de formas base potenciales.

La señal clara de tecnología de hojas, observada en el valle de SAC y la cuenca de PG para finales del Holoceno Medio, encuentra correlatos cronológicos en otras áreas de la puna argentina, como Salinas Grandes, de acuerdo con lo reportado por Jorge Fernández (1971, 1983). Particularmente, la fecha del sitio Río Grande, de ca. 5500 $\mathrm{AP}$, indicaría el momento más temprano de aparición de tecnología de hojas a escala macro-regional. A su vez, la tendencia registrada en la región del salar de Atacama, en el norte de Chile, es coherente con la destacada aquí, caracterizada por el aumento de la tecnología laminar hacia los ca. 5000 AP (Núñez et al. 2006), lo que sugiere una escala espacial amplia para el proceso de evolución de tecnología de hojas en los Andes Centro Sur.

No obstante, la evidencia de otras áreas de estudio, como Susques en la Puna de Jujuy, indican la presencia de hojas como forma base de artefactos, aunque en bajas frecuencias, desde al menos los ca. $8300 \mathrm{AP}$, como es el caso del sitio Hornillos 2, continuando este patrón hasta los $6200 \mathrm{AP}$, donde finaliza la secuencia (Hoguin 2013). Asimismo, Alero Cuevas exhibe la presencia de piezas aisladas clasificables como hojas en la capa $\mathrm{F}_{4}$, escasas pero presentes al fin, con fechas de entre 9650 y 8500 AP. Por su parte, en el sitio Abrigo Pozo Cavado, ubicado en el salar de Pocitos, también en la Puna de Salta, fueron reportados ejemplares de hojas, también en bajas frecuencias, asociados a una fecha de ca. 6200 AP (López 2013). ¿Qué implicancias tienen estas evidencias? En principio, la evidencia de Hornillos 2, único caso de los mencionados en los que se hallaron núcleos, sugiere que las hojas podrían haber sido obtenidas a partir de núcleos orientados a la obtención de lascas (Hoguin 2013), es de- 
cir, que se habría tratado de una obtención oportunista y no predeterminada. De este modo, no podemos hablar de la presencia de un sistema tecnológico de hojas para estos momentos, sino más bien de la obtención de hojas como una actividad periférica del comportamiento tecnológico, y llevada a cabo en el marco de un sistema tecnológico orientado a la obtención de lascas. Asimismo, otros contextos arqueológicos de Susques (Puna de Jujuy), aparte de Hornillos, sugieren la presencia de un sistema orientado al desbaste de hojas para finales del Holoceno Medio (Hoguin 2013), lo que es coherente y refuerza, a su vez, el patrón macro-regional. Entonces, en el contexto macro-regional, si bien se registran algunos ejemplares de artefactos formatizados sobre hojas para momentos del Holoceno Temprano e inicios del Holoceno Medio, estos estarían reflejando una obtención más bien oportunista, mientras que la señal arqueológica de un sistema tecnológico orientado a la obtención de hojas correspondería recién a finales del Holoceno Medio.

Por otra parte, y en función del segundo objetivo planteado, discutimos la dispersión espacial de los artefactos lanceolados unifaciales en la Puna de Salta. En este caso, diferentes indicadores nos permiten plantear su dispersión en el espacio a causa de la circulación de información más que de los propios objetos. En primer lugar, destacamos que los conjuntos de artefactos lanceolados unifaciales del valle de SAC y la cuenca de PG están formatizados exclusivamente sobre rocas locales de cada área. En segundo lugar, señalamos que desde el punto de vista estadístico, los conjuntos no se diferencian de manera significativa en sus medidas de longitud, ancho y espesor, y de acuerdo con el área de procedencia. En tercer lugar, en ambas áreas observamos la presencia de rasgos recurrentes como la morfología lanceolada y la reducción del espesor del bulbo mediante lascados. En conjunto, la presencia de artefactos lanceolados unifaciales formatizados en rocas de cada una de las áreas de estudio, y con similitudes métricas y morfológicas entre sí, darían mayor sustento a la hipótesis de circulación de información más que de objetos. Planteamientos similares han sido realizados para el caso de similitud métrica y morfológica entre puntas de proyectil triangulares del Holoceno Temprano, tanto de la puna argentina como de Chile (Yacobaccio 1991; de Souza 2004) así como para el caso de las puntas de proyectil "Bird", en la Patagonia (Franco et al. 2010). A su vez, la circulación de informa- ción habría implicado la transmisión de información, o transmisión cultural (Boyd y Richerson 1985; Bettinger y Eerkens 1999), involucrando el aprendizaje por imitación probablemente entre grupos distantes. En este caso, la similitud métrica y morfológica de las piezas sería la consecuencia de un "sesgo" en la transmisión de información, entendido como la replicación de un artefacto siguiendo instrucciones específicas.

Asimismo, dicha circulación de información pudo haber ocurrido en una escala espacial más amplia, considerando la presencia de artefactos lanceolados unifaciales en la Puna de Jujuy. En este caso, la información o ideas sobre cómo replicar esta clase de piezas pudo haber circulado en el marco del movimiento de otros objetos, como la obsidiana. Destacamos la presencia de la obsidiana de Zapaleri en la capa F2 de Alero Cuevas, cuya procedencia es la frontera tripartita entre Chile, Bolivia y Argentina, incluyendo la Puna de Jujuy, lo que podría implicar el contacto con grupos asentados en dicha zona. En relación a este planteamiento, será crucial el estudio de otras colecciones de artefactos lanceolados unifaciales de la puna, como las de Salinas Grandes por ejemplo, y su comparación con las del valle de SAC y la cuenca de PG.

Por otra parte, consideramos que la aparición de una señal clara de tecnología de hojas hacia los ca. 5200 AP en la Puna de Salta indica un patrón de cambio en relación a la tecnología de lascas, claramente predominante para el caso del Holoceno Temprano e inicios del Holoceno Medio. De acuerdo con lo planteado en otro trabajo (López y Restifo 2012), consideramos que la tecnología de hojas pudo haber sido una variante adecuada en un nuevo contexto, como sería el de finales del Holoceno Medio. Brevemente, señalamos que dicho contexto se habría caracterizado por un aumento de las presiones dependientes de la densidad demográfica, implicando la ocupación de aquellos espacios con recursos clave para la subsistencia como agua y camélidos, así como un aumento en el tamaño de los grupos, lo que habría dado lugar a mayores demandas energéticas (Yacobaccio y Vilá 2002; Muscio 2004; López 2008). En este marco, se plantea el desarrollo de un proceso de intensificación en la explotación de camélidos, lo que habría favorecido la obtención de un mayor retorno energético de dicho recurso (Yacobaccio 2001; Cartajena et al. 2007). 
En tal contexto, la adopción de una nueva alternativa tecnológica como la tecnología de hojas habría sido favorable por dos razones principales. En primer lugar debido a que, a diferencia de la tecnología de lascas registrada, habría sido adecuada para la obtención de formas base alargadas, útiles para la confección de diferentes artefactos con presencia de filos largos y/o puntas, destinados al procesamiento de recursos, especialmente camélidos. Si bien aún resta realizar el análisis funcional para corroborar el empleo de artefactos lanceolados unifaciales para el procesamiento de camélidos, destacamos que en la capa F2 del sitio Alero Cuevas predominan los especímenes óseos asignables a camélido, con marcas de procesamiento recurrentes y asociados a los artefactos lanceolados unifaciales (López y Restifo 2012). Sin embargo, no descartamos su posible uso también en el procesamiento de otros recursos como los vegetales, lo que profundizaremos a futuro. En segundo lugar, la tecnología de extracciones laminares habría permitido la manufactura de artefactos con diferentes clases de filos, como raederas y cuchillos, pero estandarizados, dada la regularidad métrica de las formas base obtenidas. La estandarización habría facilitado el reemplazo de piezas enmangadas, ya sea por agotamiento o rotura (Nelson 1991). La posibilidad de enmangue puede sustentarse a partir de la recurrencia de lascados en la cara ventral de los artefactos lanceolados unifaciales, rebajando el espesor del bulbo (López y Restifo 2012).

Finalmente, focalizando en el sitio Alero Cuevas, destacamos que la presencia de hojas se extiende hasta los ca. $4200 \mathrm{AP}$, fechado más tardío de la capa F2. Luego sigue un vacío de fechados de unos 2000 años, hasta llegar al segmento de ca. 2000-640 AP. En dicho segmento, los indicadores de tecnología de hojas están totalmente ausentes, destacándose las lascas como forma base, lo que también se evidencia en el sitio Quebrada Alta, ubicado a unos $5 \mathrm{~km}$ de Alero Cuevas, y con una cronología de ca. 1500 AP (López 2008; Mercuri 2011). Es relevante señalar que para estos momentos se plantea la consolidación de las estrategias económicas de producción de alimentos, como el pastoreo o la agricultura (Muscio 2004; López 2008). Además, la persistencia de la caza como estrategia complementaria (Escola 1996) habría dado lugar a la diversificación de opciones de captación de energía, minimizando el riesgo de subsistencia (López 2008). En este escenario, es probable que la tecnología de hojas ya no haya resultado una alternativa necesaria para el aumento del retorno energético de la estrategia económica global, como en el caso del proceso de intensificación en la explotación de camélidos, en el que la diversidad de estrategias de captación de energía sería menor, dependiendo principalmente de la caza. Por lo tanto, su abandono sería esperable.

\section{$\bullet$ Conclusiones}

El estudio de la tecnología de hojas en la puna constituye una temática que puede ser abordada a través de múltiples ejes de análisis. En este trabajo destacamos dos de ellos. En primer lugar destacamos la temática de la aparición de la tecnología de hojas en la Puna de Salta, cuya señal arqueológica se manifiesta a partir de los ca. $5200 \mathrm{AP}$, extendiéndose hasta los $4200 \mathrm{AP}$, es decir, el segmento de finales del Holoceno Medio, y comienzos del Holoceno Tardío. Esto es coherente con los hallazgos de Salinas Grandes y Susques, en la Puna de Jujuy (Fernández 1983; Hoguin 2013). Asimismo, destacamos su ausencia a partir de los ca. 2000 AP. Sin embargo, es necesario profundizar la investigación en el segmento de los $3000 \mathrm{AP}$, para dar firmeza a dicha fecha, lo que constituye una próxima etapa de trabajo. Por su parte, los contextos del Holoceno Temprano e inicios del Holoceno Medio, si bien presentan ejemplares aislados de hojas, no estarían dando cuenta de un sistema tecnológico orientado a tal fin.

En segundo lugar abordamos la dispersión espacial de la tecnología de hojas, a partir de los artefactos lanceolados unifaciales. Es posible que la circulación en el espacio de la tecnología de hojas haya implicado alguna clase de contacto entre poblaciones distantes, al menos considerando una mesoescala espacial, abarcando las áreas del valle de SAC y la cuenca de PG. En este sentido, será relevante aumentar las muestras para replicar el análisis estadístico realizado, así como incluir nuevas muestras de otras áreas de la Puna de Salta, como el sector salteño de Salinas Grandes (véase Patané Aráoz 2013), así como de la Puna de Jujuy.

Por último, consideramos que tanto la aparición como la dispersión de hojas en la puna son dos temáticas que contribuyen a la comprensión del proceso de evolución cultural de la macro-región, pero no son las únicas. En 
este sentido, la temática de la declinación de la tecnología de hojas merece mayor profundización. Tanto el abordaje de esta temática, como la continuidad en los dos ejes priorizados en este trabajo, sumados a la propuesta de nuevas vías de investigación, favorecerán la comprensión de un proceso que aún no ocupa un rol central, pero que reviste total importancia para la comprensión del proceso de evolución cultural en las tierras altas de los Andes Centro Sur.

Agradecimientos A las comunidades de San Antonio de los Cobres y Santa Rosa de los Pastos Grandes, por recibirnos siempre con los brazos abiertos en cada campaña de trabajo. Al Doctor Rodolphe Hoguin por su lectura. A la Licenciada Daniela S. Cañete Mastrángelo por su ayuda en el tratamiento digital de las imágenes.

\section{* Referencias citadas}

ASCHERO, C. 1975. Ensayo para una clasificación morfológica de artefactos líticos. Informe de avance presentado a CONICET. Ms.

1983. Ensayo para una clasificación morfológica de artefactos líticos. Cátedra de Ergología y tecnología, Universidad de Buenos Aires.

ASCHERO, C. y S. HOCSMAN. 2011. Arqueología de las ocupaciones cazadoras-recolectoras de fines del Holoceno Medio de Antofagasta de la Sierra (Puna Meridional Argentina). Chungara 43:393-411.

ASCHERO, C. A., L. MANZI y A. GÓMEZ. 1993. Producción lítica y uso del espacio en el nivel 2b4 de Quebrada Seca 3. Relaciones de la Sociedad Argentina de Antropología XIX: 191-213.

BAR-YOSEF, O. y S. KUHN. 1999. The big deal about blades: Laminar technology and human evolution. American Anthropologist 101: 322-328.

BELLELLI, C. y H. NAMI. 1994. Hojas, experimentos y análisis de desechos de talla. Implicaciones arqueológicas para la Patagonia Centro-Septentrional. Cuadernos del INAPL 15: 199-224.

BETTINGER, R. y J. EERKENS. 1999. Point typologies, cultural transmission, and the spread of bow and arrow technology in the prehistoric Great Basin. American Antiquity 64(2): 231-242.

BOËDA, E. 1997. Technogenèse des systèmes de production lithique au Paléolithique inférieur et moyen en Europe occidentale et au Proche-Orient. Tesis de habilitación para dirección de investigadores. Universidad de París X, Nanterre.

BOYD, R. y P. RICHERSON. 1985. Culture and the evolutionary process. University of Chicago Press, Chicago.

CARDILLO, M. 2002. Transmisión cultural y persistencia diferencial de rasgos. Un modelo para el estudio de la variación morfológica de las puntas de proyectil lanceoladas de San Antonio de los Cobres, Provincia de Salta, Argentina. En Perspectivas integradoras entre Arqueología y Evolución, G. Martínez y J. Lanata (Eds.), pp. 97-119. INCUAPA, Universidad Nacional del Centro de la Provincia de Buenos Aires, Buenos Aires.

CARTAJENA, I., L. NÚÑEZ y M. GROSJEAN. 2007. Camelid domestication on the western slope of the Puna de Atacama, northern Chile. Anthropozoologica 42(2): 155-173.

COLLARD, M., B. BUCHANNAN, A. TUTTLR y M. O'BRIEN. 2012. Niche construction and the toolkits of hunter gatherers and food producers. Biological Theory 6: 251-259.

DE SOUZA, P. 2004. Cazadores recolectores del Arcaico Temprano y Medio en la Cuenca del río Loa: sitios, conjuntos líticos y sistemas de asentamiento. Estudios Atacameños 27: 7-44.

ESCOLA, P. 1996. Riesgo e incertidumbre en economías agropastoriles: Consideraciones teórico-metodológicas. Arqueología 6: 9-23.

FERNÁNDEZ, J. 1971. La Edad de la Piedra en la Puna de Atacama (una investigación regional y cronológica, una aportación de la ciencia geográfica a la solución del problema vinculado a la temprana instalación humana en Sudamérica). Revista del Instituto de Antropología, serie 3, Tucumán.

1983. Río Grande. Exploración de un centro precerámico en las altas montañas de Jujuy, Argentina. Ampurias 45/46: 54-83.

FERNÁNDEZ DISTEL, A. 1978. Nuevos hallazgos precerámicos en la región de Salinas Grandes, Puna de Jujuy, Argentina. Revista del Instituto de Antropología VI: 15- 62.

2007. El yacimiento de Guayatayoc (Jujuy, Argentinas): Sus materiales líticos y un fechado de radiocarbono inédito. Cuadernos de la Universidad Nacional de Jujuy 32: 151-166.

FRANCO, N., J. GÓMEZ OTERO, G. GURÁIEB, S. GOYE, N. CIRIGLIANO y A. BANEGAS. 2010. Variaciones espaciales en diseños de puntas pedunculadas medianas en Patagonia argentina: una nueva aproximación. En Arqueología argentina en el bicentenario de la revolución de Mayo, R. Bárcena y $\mathrm{H}$. Chiavazza (Eds.), pp. 283-287. Universidad Nacional de Cuyo, Mendoza. 
HAIR, J., W. BLACK, B. BABIN y R. ANDERSON. 2005. Multivariate Data Analysis. Pearson Prentice Hall, Upper Saddle River.

HAMMER, Ø., D. HARPER y P. RYAN. 2001. PAST: Paleontological statistics. Http://www.toyen.uio.no/ ohammer/past.

HOGUIN, R. 2013. Evolución y cambios técnicos en sociedades cazadoras-recolectoras en la Puna Seca de los Andes Centro-Sur. Tesis de doctorado. Facultad de Filosofía y Letras, Universidad de Buenos Aires. Ms.

KUHN, S. 2006. Trajectories of Change in the Middle Paleolithic of Italy. En Transitions before transitions. Evolution and stability in the Middle Paleolithic and Middle Stone Age, E. Hoversa y S. Kuhn (Eds.), pp. 109-120. Springer, New York.

LÓPEZ, G. 2008. Arqueología de cazadores y pastores en tierras altas. Ocupaciones humanas a lo largo del Holoceno en Pastos Grandes, Puna de Salta, Argentina. BAR international series, Oxford, Inglaterra.

2013. Ocupaciones humanas y cambio a lo largo del Holoceno en abrigos rocosos de la Puna de Salta, Argentina: Una perspectiva regional. Chungara. Revista de antropología chilena 45(3): 411426.

LÓPEZ, G. y F. RESTIFO. 2012. The Middle Holocene domestication and intensification of camelids in north Argentina, tracked by zooarchaeology and lithics. Antiquity 86: 1041-1054.

MERCURI, C. 2011. Variabilidad de estrategias tecnológicas liticas durante el período Formativo (ca. 2400-1400 AP) en la Puna de Salta. Tesis Doctoral. Facultad de Filosofía y Letras, Universidad de Buenos Aires. Ms.

MERCURI, C. y F. RESTIFO. 2011. Application of physico-chemical and macroscopic methods to lithic artifact studies from Alero Cuevas site (Salta, República Argentina): A complementary approach. En Physical, chemical and biological markers in argentine archaeology: theory, methods and applications, D. M. Kligmann y M. R. Morales (Eds.). BAR international series, Archaeopress, Oxford (en prensa).

MUSCIO, H. 2004. Dinámica Poblacional y Evolución Durante el Período Agroalfarero Temprano en el Valle de San Antonio de los Cobres, Puna de Salta, Argentina. Tesis Doctoral. Facultad de Filosofía y Letras, Universidad de Buenos Aires. Ms.

2011. Ocupaciones humanas a cielo abierto de finales del Holoceno Medio y comienzos del Holoceno Tardío en el Valle de San Antonio de los Cobres, Puna de Salta. Comechingonia 15: 171-190.

NELSON, M. 1991. The study of technological organization. Archaeological Method and Theory 3: 57-100.
NÚÑEZ, L., M. GROSJEAN e I. CARTEJENA. 2006. Ocupaciones Humanas y Paleoambientes en la Puna de Atacama. Universidad Católica del Norte, Taraxacum, San Pedro de Atacama.

PATANÉ ARÁOZ, J. 2013. Prospecciones arqueológicas en Salinas Grandes (Departamento La Poma, Provincia de Salta) y reporte de una punta "cola de pescado". Relaciones de la Sociedad Argentina de Antropología 38(1): 247-255.

QUINTERO, L. y P.WILKE. 1995. Evolution and economic significance of Naviform core and blade technology in the southern Levant. Paleorient 21: 17-33.

RESTIFO, F. 2013. Tecnología de caza durante el Holoceno Temprano y Medio en la Puna de la Provincia de Salta (República Argentina): Patrones de variación y procesos de cambio. Comechingonia 16:57-82.

RESTIFO, F. y R. HOGUIN. 2012. Risk and technological decision-making during the early to mid-Holocene transition: A comparative perspective in the Argentine Puna. Quaternary international 256:35-44.

RUTHSATZ, B. y C. MOVÍA. 1975. Relevamientos de las estepas andinas del noreste de la Provincia de Jujuy, República Argentina. FECIC, Buenos Aires.

SCHOBINGER, H., R. BÁRCENAy J. FERNÁNDEZ. 1974. Excavación arqueológica del sitio precerámico "Espinazo del Diablo", Mina Aguilar, provincia de Jujuy. Relaciones 8: 89-99.

TIXIER, J. 1963. Typologie de l'Epipaléolithique du Maghreb. M'emoires du Centre de Recherches Anthropologiques, Préhistoriques et Ethnographiques, 2. Alger, París.

TURNER, J. C. 1964. Descripción geológica de la Hoja 7 c - Nevado de Cachi. Dirección Nacional de Minería y Geología, Buenos Aires.

VILELA, C. 1969. Descripción geológica de la hoja 6C, San Antonio de los Cobres. Dirección Nacional de Minería y Geología, Buenos Aires.

YACOBACCIO, H. D. 1991. Sistemas de Asentamiento de los Cazadores-Recolectores Tempranos en los Andes Centro Sur. Tesis de Doctorado. Universidad de Buenos Aires. Ms.

2001. La domesticación de camélidos en el Noroeste Argentino. En Historia Argentina Prehispánica, I, E. E. Berberián y A. E. Nielsen (Eds.), pp. 7-40. Editorial Brujas, Córdoba.

YACOBACCIO, H. D. y B. VILÁ. 2002. Condiciones mecanismos y consecuencias de la domesticación de camélidos. Estudios Sociales del NOA 3: 4-27. 